\title{
USES AND FALLACIES OF INTRACARDIAC ELECTROGRAMS AT THE PULMONARY VALVE*
}

\author{
BY
}

\author{
K. K. DATEY AND M. J. GANDHI
}

From the Departments of Cardiology and Medicine, K.E.M. Hospital and G.S. Medical College, Bombay, India

Received March 22, 1961

Intracardiac electrograms by cardiac catheterization were first recorded by Lenegre and Maurice (1945). The electrographic patterns in various chambers of the heart were studied by Hecht (1946), Battro and Bidoggia (1947), and Kossmann et al. (1950), and were mainly of value in studying the activation of the heart. Their importance during cardiac catheterization was first shown by Emslie-Smith (1955), who also suggested their usefulness in the exact localization of pulmonary stenosis (Emslie-Smith et al., 1956). Although various authors have studied the patterns of the intracardiac electrogram in the great vessels and in the different chambers of the heart, no systematic study of the changes occurring at the pulmonary valve has been presented except by Emslie-Smith et al. (1956) and Kossmann et al. (1950), who studied these changes in normal subjects without simultaneous pressure tracings.

This study was therefore undertaken to analyse the variations in the intracardiac electrograms at the site of the pulmonary valve and in the outflow tract of the right ventricle, and to determine their value in the exact localization of obstruction in the outflow of the right ventricle.

\section{MATERIALS AND MethodS}

Twenty-seven cases of heart disease of different ætiologies were studied. They were (1) acquired heart disease (11 cases), (2) pulmonary stenosis (simple or complicated) (4 cases), and (3) other congenital heart diseases (12 cases).

Cardiac catheterization was carried out in the supine position under fluoroscopic control, a standard electrode catheter (U.S. Catheter Corporation-size 6 to $8 \mathrm{~F}$, length $100 \mathrm{~cm}$.) being used. The catheter was introduced through a medial antecubital vein and was negotiated into the pulmonary trunk. A withdrawal tracing across the pulmonary valve was recorded, with simultaneous registration of the pressures and of an intracardiac electrogram on a double channel direct-writing machine, at a paper speed of 25 or $100 \mathrm{~mm}$./sec. The withdrawal was effected slowly under fluoroscopic control so as to be certain that the tip of the catheter moved very slowly across the pulmonary valve. In case of a sudden movement of the catheter tip the procedure was repeated. Usual precautions were taken to avoid AC interference, e.g. switching off other circuits in the room, manipulating lamp plugs, etc., but the catheter tip was not earthed in any of our cases.

As the proximal end of the metal electrode was $2 \mathrm{~mm}$. from the terminal opening, it was postulated that in the withdrawal tracings from the pulmonary trunk to the right ventricle, the change in the intracardiac electrogram might precede the pressure change. This, however, did not occur. In most cases the descending limb of the last pulmonary artery pressure pulse was continued to zero level and this was succeeded by a change in both tracings (Fig. 1 and 2). For the purpose of this study, the intracardiac electrograms corresponding to the last pulmonary trunk and the first right ventricular pressure pulses were used for comparison. The last few beats from the pulmonary trunk tracing and the first few from the right ventricle,

* Paper presented at the Sixth International Congress on Diseases of the Chest, Vienna, August, 1960. 
in the vicinity of the pulmonary valve, were also studied with reference to the following points-(a) changes in $\mathrm{P}$ waves, (b) alterations in the QRS complexes, both in voltage and pattern, and (c) appearance of ventricular extrasystoles and/or arrhythmias, etc.

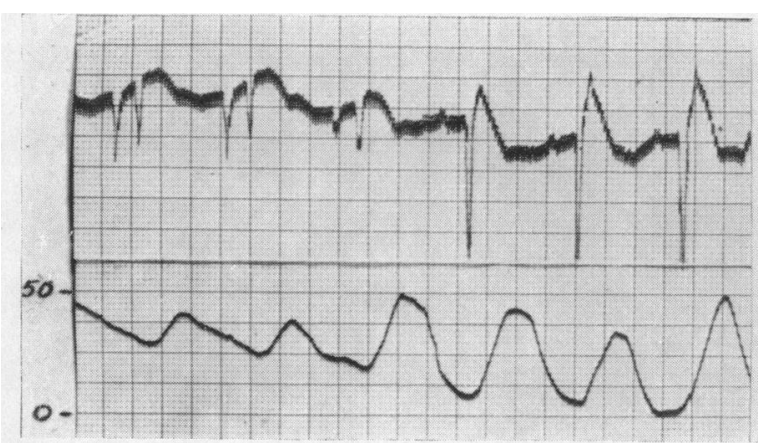

FIG. 1.--Inverted $\mathbf{P}$ changes into upright $\mathrm{P}$. Sudden increase of voltage of QRS complex. The descending limb of last P.A. pressure pulse is continued to zero level with simultaneous change in the intracardiac electrogram. Pressure scale in $\mathrm{mm}$. $\mathrm{Hg}$ in all figures.

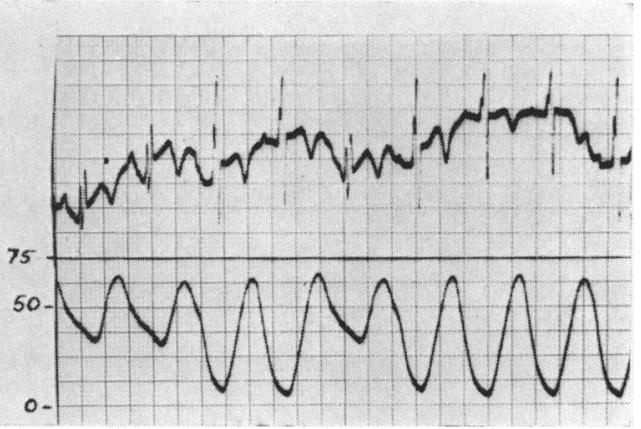

FIG. 2.-Change in intracardiac electrogram and pressure tracing due to fluctuation of electrode across the pulmonary valve.

In this and other figures the intracardiac electrogram is above and the pressure curve below.

\section{RESULTS}

As the electrode catheter was withdrawn from the pulmonary trunk to the right ventricle, changes were noted in the intracardiac electrogram at the site of the pulmonary valve, and these are described for the $\mathrm{P}$ waves and for the QRS complexes.

(A) P Waves

\section{Pulm. trunk}

(1) Inverted became Inverted became Inverted showed

(2) Upright remained

(3) Iso-electric remained

\section{Rt. ventricle}

\section{Upright}

Biphasic

Delayed change

Upright

Iso-electric
In 8 tracings (Fig. 1 and 3)

In 2 tracings (Fig. 4)

In 1 tracing

In 4 tracings (Fig. 5)

In 12 tracings

Thus changes in $\mathrm{P}$ waves are present both before and after the valve is negotiated.

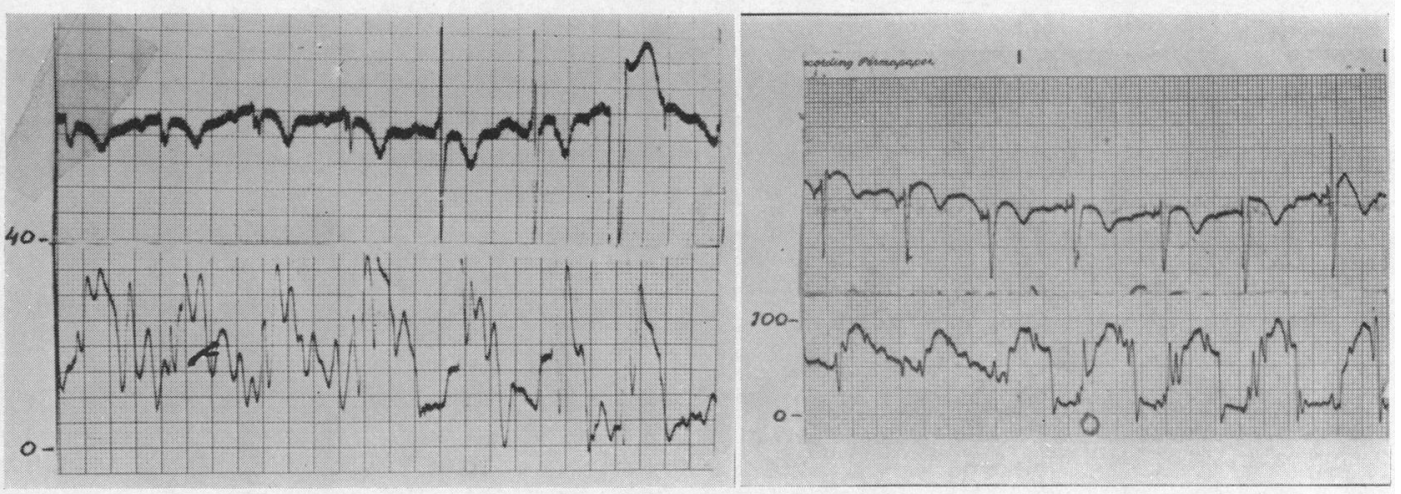

FIG. 3.-Sudden change in the configuration of the $\mathrm{P}$ wave.

FIG. 4.- Inverted $P$ changes to biphasic $P$. There is a delayed change in the voltage of QRS. 
(B) QRS CoMPLEXES

(1) Voltage. (a) A sudden increase in the voltage near the valve was seen in 16 cases (Fig. 1, 2), but in 2 there was a further increase of voltage in the right ventricle after a few beats.

(b) A sudden increase in the voltage of $\mathbf{R}^{\prime}$ but a gradual one in that of $\mathbf{S}^{\prime}$ was present in 3 cases starting from the last two beats in the pulmonary trunk and continuing for about seven beats in the right ventricle (Fig. 6).

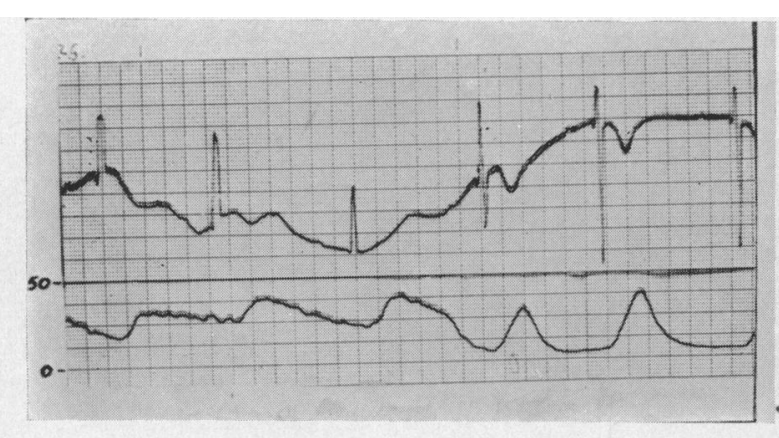

Fig. 5.-P upright in both right ventricle and pulmonary trunk. qR changing to $\mathrm{qRS}$.

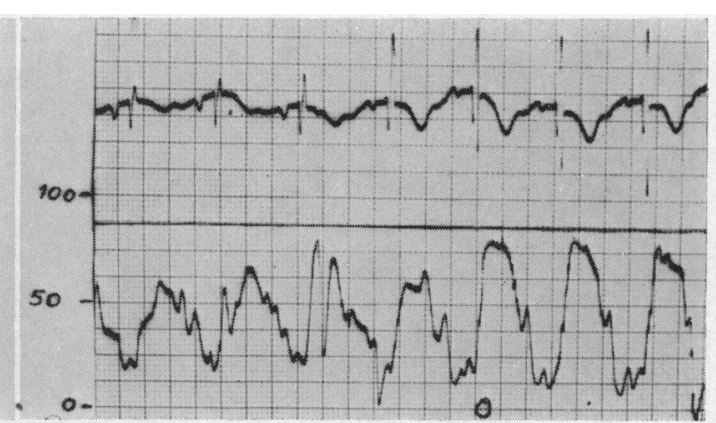

FIG. 6.-Gradual increase in the voltage of QRS. Relatively sudden increase in the magnitude of $R^{\prime}$ and a gradual increase of $\mathbf{S}^{\prime}$.

(c) A delay in the increase of voltage, at the site of the pulmonary valve, was found in three cases, the change in voltage occurring from the 3rd to the 6th beat in the ventricle (Fig. 4).

(d) In two cases there was no change (Fig. 7).

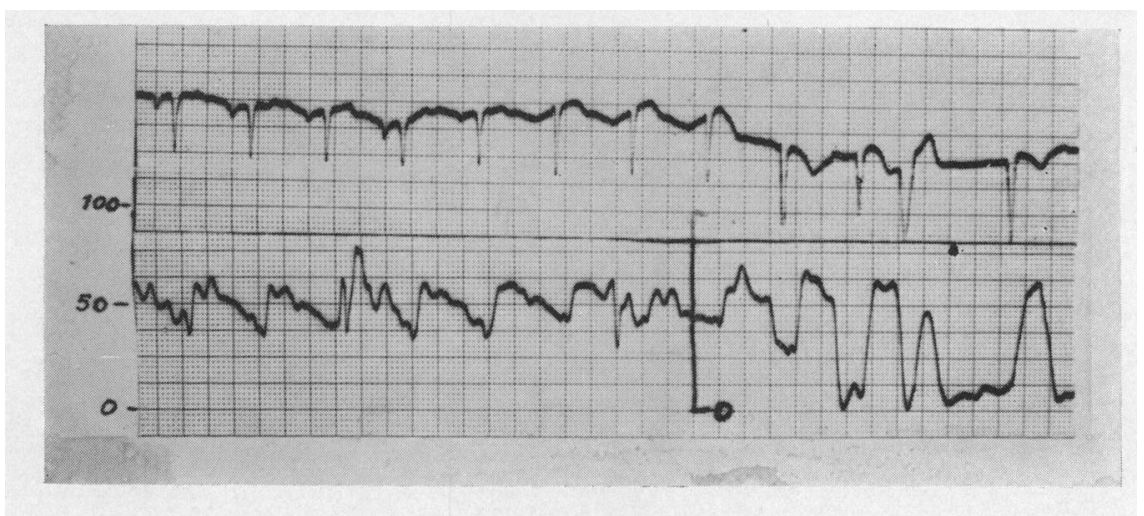

FIG. 7.-No significant change in the voltage of QRS.

(2) Multiple Ventricular Premature Beats. Ventricular premature beats of large deflections or ventricular arrhythmias (Fig. 8) occurred in 3 cases as soon as the catheter was withdrawn into the ventricle. This is usually due to the catheter suddenly whipping into the cavity and pressing on the endocardium of the right ventricle.

(3) QRS Patterns. The changes in the QRS pattern depended upon the original pattern in the pulmonary trunk.

(a) $r S$ pattern in pulmonary trunk. The pattern remained the same in 6 cases with or without some increase in the voltage of $\mathrm{r}$ and/or $\mathrm{S}$ (Fig. 1). The notch on the ascending limb of 
$\mathrm{S}$, when present, disappeared. In one case two intermediate beats with $\mathrm{Q}$ waves appeared. The pattern changes to $r s R^{\prime} S^{\prime}$ in two and to $Q R S$ in one. In one a late $r$ appeared. In only two of these cases was the $T$ wave upright in the right ventricle, the pattern being QRS and rsR'S'.

(b) $R S R^{\prime} S^{\prime}$ pattern or its variants. This pattern in the pulmonary trunk was seen in 5 cases and in these various changes occurred. It remained the same in one. In another it changed to $R R^{\prime} S$. In two, late $R$ and $S$ increased considerably in voltage and in one of these $R^{\prime \prime}$ appeared even though the QRS interval did not widen. In one, the rsr's' pattern changed to RSr's' with widening of the QRS interval from $0.08 \mathrm{sec}$. to $0 \cdot 12 \mathrm{sec}$.

(c) $q R s$ or $q R$ pattern. This was present in 4 cases. The $\mathrm{S}$ wave appeared or persisted in the right ventricular cavity electrogram (Fig. 5).

(d) $r s r^{\prime}$ pattern. This appeared in 4 cases. $\mathrm{r}^{\prime}$ appeared as a notch on the ascending limb of $s$ wave or became $R^{\prime}$. In two it changed to rsR' $S^{\prime}$. However, in one this change was delayed and occurred from the fourth beat onwards, in the right ventricle.

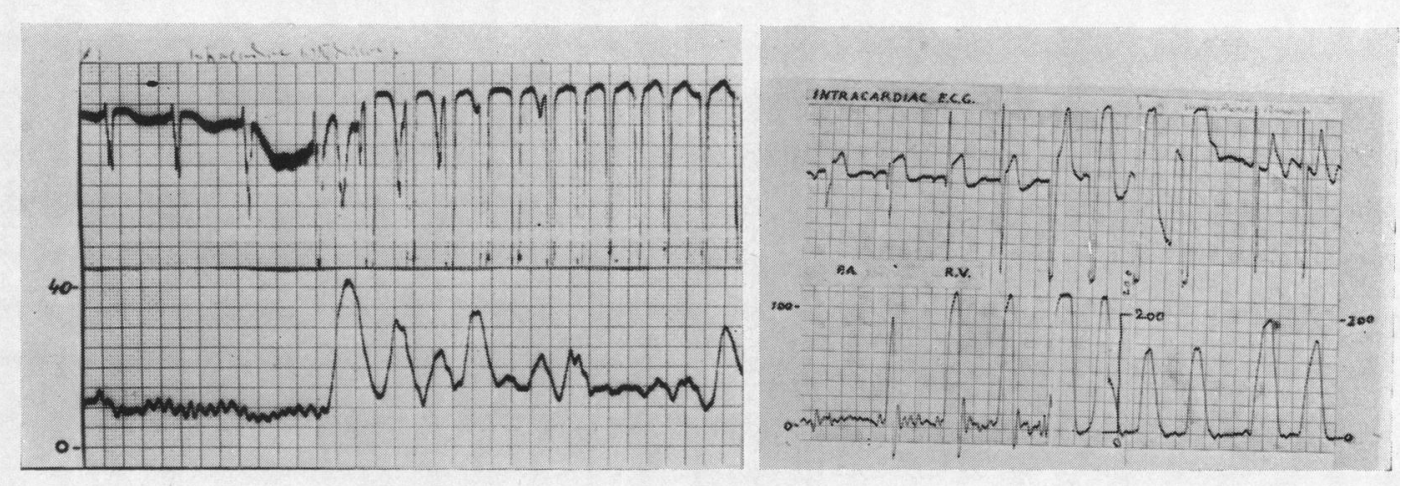

FIG. 8.-Development of ventricular tachycardia.

FIG. 9.-Typical intracardiac electrogram and pressure tracing in a case of pulmonary valvular stenosis confirmed by surgery. Pressure scale in P.A. and R.V. is initially $100 \mathrm{~mm}$. and is then changed to $200 \mathrm{~mm}$. $\mathrm{Hg}$ in the latter half of the tracing.

\section{Discussion}

A diagnosis by means of pressure tracings of the level of obstruction at the outflow tract of the right ventricle, whether it be valvular, infundibular, or combined, has certain fallacies. Connolly et al. (1953) pointed out that if infundibular stenosis is mild and valvular stenosis severe, no zone of intermediate pressure may be detected, as the drop in pressure from the right ventricle proper to the infundibular region depends on the degree of infundibular stenosis relative to that present at the valve. Similarly, in an infundibular stenosis with regurgitation at the pulmonary valve, as in bacterial endocarditis, the pressure patterns will simulate those of valvular stenosis. Moreover, misleading infundibular patterns may appear as a result of the Venturi effect (Sobin et al., 1954). In view of these pitfalls reliance cannot be placed on the pressure tracings alone for the site of the obstruction. Hence Emslie-Smith et al. (1956) advocated, as a further aid, the use of an intracardiac electrogram across the pulmonary valve. Fig. 9 shows a typical tracing in a case of valvular stenosis. In view of its importance, it is imperative to know the variations occurring in the intracardiac electrogram across the pulmonary valve.

Changes in $P$ Waves. Although in the majority of cases when the $P$ wave is inverted in the pulmonary trunk, it becomes upright at the site of the valve, this change may also be noted both before or after the valve is negotiated. This is not surprising in view of the fact that the configuration of the $\mathbf{P}$ wave at a particular location will depend upon the relation of the electrode to the 
atrium; when it is at the caudal end of the atrium, there will be an upright $\mathrm{P}$ wave, when in the cephalad location there will be an inverted $\mathbf{P}$. Moroever, $\mathbf{P}$ is isoelectric in many cases. Hence $\mathbf{P}$ wave changes are not reliable criteria for the location of the site of the pulmonary valve.

Changes in the QRS Complex. (1) Voltage. The change in the voltage of the QRS complex is supposed to be the most important single criterion for the localization of the site of the pulmonary valve. In this series the change in voltage occurred suddenly (Fig. 1,2) as well as gradually (Fig. 4). It is clear that whenever the change in the voltage across the pulmonary valve is gradual, the site of the pulmonary valve cannot be localized with certainty from an intracardiac electrogram. In some cases, the change in voltage did not occur at the pulmonary valve, but manifested itself a little later. If this change in the voltage coincides with the entry of the catheter from the infundibulum to the right ventricle, the infundibular tracing might be mistaken for a pulmonary trunk tracing and thus an infundibular stenosis might masquerade as a valvular one.

An increase in voltage may be expected when the exploring electrode is near the source of the vector. The QRS complex according to Sodi-Pallares (1956) is composed of three main vectors, viz.: (1) activation of the interventricular septum and some apical portion of the free walls of the right ventricle; (2) activation of the free walls of both ventricles but mainly those of the left; and (3) activation of the basal cardiac mass (i.e. basal regions of both ventricles and interventricular septum).

The increased voltage may be due to a greater magnitude of one or all of these vectors. According to Lewis's concept, the upper part of the interventricular septum on its left surface is the first to be activated, the impulse then spreading from above downwards. Hence the first vector should be predominant near the upper muscular portion of the interventricular septum, which is a little below the level of the pulmonary valve. However, Sodi-Pallares et al. (1947) believe that the activation of the septum spreads from below upwards. The earliest septal vector is directed from left to right and points towards the trabecular zone and apex of the right ventricle and is therefore most prominent in this zone. The next septal vector is directed upwards and points to the basal regions adjacent to the tricuspid and pulmonary valves: this vector presents as a late $r$ at the right upper septal surface. Thus the concepts of Lewis and of Sodi-Pallares et al. (1947) regarding the activation of the interventricular septum are at variance.

Similarly, the second vector will be prominent and become more so as the mid-right ventricle is entered. The third vector is greatest just at the outflow tract and will disappear as the mid-right ventricle is entered. At the same time, as the proximal part of the electrode is at a distance of $2 \mathrm{~mm}$. from the opening in the catheter, there is a slight time lag between the pressure and tracing of electrical events. Thus, as multiple factors are involved it is not surprising that many variations in voltage occur.

(2) Appearance of Multiple Premature Beats. The entry of the tip of a cardiac catheter into the right ventricle, whether from the right atrium or pulmonary trunk, is often heralded by the development of premature beats, due to pressure of the catheter tip on the endocardial surface. Since these premature beats are of right ventricular origin their configuration is that of the QS type. Kossmann et al. (1950) observed the appearance of atrial premature beats in one case and ascribed them to the pressure of the proximal part of the catheter on the right atrium. This was not seen in any of our cases.

(3) Changes in the Pattern. As the electrode catheter is withdrawn from the pulmonary trunk to the right ventricle, various changes in QRS pattern appear. At the outflow tract of the right ventricle, the vector due to the activation of the upper part of the septum or crista supraventricularis causes $\mathbf{R}^{\prime}$, while activation of the free wall of the right ventricle below the pulmonary and tricuspid valve causes $S^{\prime}$. Hence as the electrode moves from the pulmonary trunk to the right ventricle, both late $\mathbf{R}$ and $\mathbf{S}$ may increase in magnitude, if already present. This vector due to the activation of the upper part of the septum or crista supraventricularis may appear as a notch on the ascending limb of $\mathrm{S}$ in the tracing in the pulmonary trunk. It may completely disappear if it is overwhelmed by the vectors reflecting the depolarization of the free walls of both the ventricles or it may change 
to $\mathbf{R}^{\prime}$. Sometimes $\mathbf{R}^{\prime}$ is so predominant that it masks the vector due to the activation of the free walls of the left ventricle, thus changing the $\mathbf{r S R}^{\prime} \mathbf{S}^{\prime}$ pattern to $\mathrm{rR}^{\prime} \mathrm{S}$. However, it is difficult to explain the disappearance of a small late $r$ and its reappearance as a late $R$, after a few beats in the right ventricle during withdrawal from the pulmonary valve to the right ventricle.

In one case, $\mathbf{R}^{\prime \prime}$ appeared even though the QRS interval was normal. Dickens and Goldberg (1958) have described a similar endocardial $R^{\prime \prime}$ in complete right bundle-branch block. They have pointed out that this $R^{\prime \prime}$ or $r^{\prime \prime}$ corresponds to $R^{\prime}$ or $r^{\prime}$ of V4R and therefore to the usual endocardial $\mathbf{R}^{\prime}$ also observed in other cases. They ascribe the intermediate $r^{\prime} s^{\prime}$ to depolarization of the middle portion of the septum, which is normally obscured by a predominantly negative field created by depolarization of the free ventricular walls. However, $\mathbf{R}^{\prime \prime}$ does not necessarily correspond to the usual endocardial $R^{\prime}$.

The appearance of qRS and qR patterns in the pulmonary trunk and the right ventricle is difficult to explain. Whether this q is related to the activation of the lower one-third of the septum or whether it is due to activation of the free wall of the left ventricle is not clear. Simultaneous leads may be of help here. In the former instance, a q wave may be due to orientation of the electrode in the pulmonary artery to the cavity of the left ventricle. A q wave in the right ventricle may also be due to the contact of the exploring electrode with the septal surface (Sodi-Pallares and Calder, 1956).

\section{SUMMARY AND CONCLUSIONS}

Twenty-seven cases of heart disease of different ætiologies were studied to determine the changes in the intracardiac electrogram at the pulmonary valve.

As the electrode catheter was withdrawn from the pulmonary trunk to the right ventricular outflow tract, the following changes were noted.

Changes in $P$ wave. An inverted $P$ wave in the pulmonary trunk became upright at the site of the valve in most cases, but in some this change was noted either before or after the pulmonary valve was negotiated.

Changes in QRS complex. Although sudden increase in the voltage of QRS was seen in most cases, in others the change was gradual or delayed, and in some no change occurred. Different changes occurred in the pattern, depending on the initial pattern in the pulmonary trunk. In general, late $\mathbf{R}$ and $\mathbf{S}$ waves appeared or increased in magnitude, and in some, $\mathbf{R}^{\prime \prime}$ was noted. In some cases the changes were masked by the development of ventricular premature beats and/or arrhythmias.

The probable mechanism of these changes has been discussed.

The value of the intracardiac electrogram and its fallacies in the localization of the site of obstruction, whether in the pulmonary valve or in the right ventricular outflow tract, have been analysed.

The study of intracardiac electrographic patterns across the pulmonary valve suggests that it would serve as a useful aid in the localization of the site of obstruction at the outflow tract of the right ventricle, particularly because the infundibular hypertrophy will exaggerate the normal changes. However, the variations in these changes and their fallacies must be borne in mind.

\section{REFERENCES}

Battro, A., and Bidoggia, H. (1947). Amer. Heart J., 33, 604.

Connolly, D. C., Lev, R., Kirklin, J. W., and Wood, E. H. (1953). Proc. Staff Meet. Mayo Clinic, 28, 65.

Dickens, J., and Goldberg, H. (1958). Amer. Heart J., 56, 8 and 372.

Emslie-Smith, D. (1955). Brit. Heart J., 17, 219.

, Lowe, K. D., and Hill, I. G. W. (1956). Brit. Heart J., 18, 29.

Hecht, H. H. (1946). Amer. Heart J., 32, 39.

Kossmann, C. E., Berger, A. R., Rader, B., Brumlik, J., Briller, S. A., and Donnelly, J. H. (1950). Circulation, $2,10$.

Lenegre, J., and Maurice, P. (1945). Arch. Mal. Caeur., 38, 298.

Levine, H. D., Hellems, H. K., Wittenborg, M. H., and Dexter, L. (1949). Amer. Heart J., 37, 46.

- Dexter, L., and Tucker, A. S. (1949). Amer. Heart J., 37, 64.

Sobin, S. S., Carson, M. J., Johnson, J. L., and Baker, C. R. (1954). Amer. Heart J., 48, 416.

Sodi-Pallares, D., Vizcaino, M., Soberson, J., and Cabrera, C. E. (1947). Amer. Heart J., 33, 819.

and Calder, R. M. (1956), New Basis of Electrocardiography. The C. V. Mosby Company, St. Louis. 